\title{
Obtaining Accurate $p$ Values from a Dense SNP Linkage Scan
}

\author{
William C.L. Stewart ${ }^{a, b}$ Ryan L. Subaran ${ }^{a}$ \\ a Battelle Center for Mathematical Medicine, Research Institute at Nationwide Children's Hospital, and \\ ${ }^{b}$ Department of Pediatrics, Ohio State University, Columbus, Ohio, USA
}

\section{Key Words}

Type 1 error $\cdot$ Linkage analysis · Next-generation

sequencing $\cdot$ Linkage disequilibrium

\begin{abstract}
A major concern of resequencing studies is that the pathogenicity of most mutations is difficult to predict. To address this concern, linkage (i.e. co-segregation) analysis is often used to exclude neutral mutations and to better predict pathogenicity among the candidate mutations that remain. However, when linkage disequilibrium (LD) is present in the population but ignored in the analysis, unlinked regions with high LD can inflate the type 1 error and thousands of neutral mutations may be mistakenly included in a follow-up resequencing study, which could dramatically reduce the power to identify causal variants. To illustrate the need for concern, we simulated data on a sparsely spaced panel of single nucleotide polymorphisms (average spacing $1.27 \mathrm{cM}$ ) using an LD pattern estimated from real data. In our simulations, we find that the type 1 error of the maximum LOD can be as high as $14 \%$. Therefore, to control the type 1 error of linkage tests we created Haplodrop - a fast and flexible simulation program that generates the haplotypes of founders with LD and then 'drops' these haplotypes with recombination to all non-founders in the pedigree. Haplodrop can be used to control the type 1 error of any linkage test, agrees
\end{abstract}

\section{KARGER \\ Fax +41613061234 \\ E-Mail karger@karger.ch}

www.karger.com
(C) 2012 S. Karger AG, Basel

$0001-5652 / 12 / 0741-0012 \$ 38.00 / 0$

Accessible online at:

www.karger.com/hhe well with existing software, accommodates arbitrary pedigree structures, and scales easily to the whole genome. Moreover, by correctly excluding mutations that lie in unlinked regions with high LD, Haplodrop should aid significantly in reducing the multiple testing burden of follow-up resequencing studies.

Copyright $\odot 2012$ S. Karger AG, Basel

\section{Introduction}

The use of current sequencing technologies as a tool for finding causal variants beneath linkage peaks holds considerable promise for the identification of genetic factors with clinically meaningful effects $[1,2]$. However, the overall efficacy of this approach is greatly reduced if the probability of resequencing clinically unimportant variants is too high. For several commonly used next-generation sequencing strategies, the primary determinant of this probability is the type 1 error of an earlier linkage study. However, the type 1 error of many linkage studies is inflated, especially when the genotypes of founders are missing, due to the fact that most multipoint linkage methods ignore the non-random distribution of alleles in

W.C.L.S. and R.L.S. contributed equally to this work. 
the population (i.e. linkage disequilibrium, LD). Worse yet, the inflation can be severe when the families are enriched for affected members (e.g. affected sibling pairs, ASPs) [3, 4]. For example, using data simulated on 1,000 ASPs under the null hypothesis of no linkage but in the presence of high LD, Huang et al. [4] showed that the maxLOD exceeded 9.

Another difficulty with controlling the type 1 error of a multipoint linkage test (especially when LD is present) is that the null distribution is usually unknown. In the absence of a known distribution, researchers used specific thresholds to assess the significance of the maximum LOD. However, these thresholds range from 3.0 to 3.6 [5], which makes type 1 error a moving target. An interesting exception to this general rule is the maximum nonparametric linkage statistic [6]. In the presence of LD, the $\mathrm{p}$ value for this test can be approximated analytically with little or no bias provided that one uses the computationally intensive 'haplotype-block'-based method of Abecasis and Wigginton [7].

As for existing methods that seek to control the type 1 error in the presence of LD, they tend to be either operationally demanding, limited in scope, or suboptimal in terms of power. For example, the program Genedrops [8, 9] required the use of Makeped (or its equivalent), Java, and numerous tuning parameters with little or no guidance as to what values might be appropriate for the simulation of interest. Furthermore, the documentation is sparse and, at times, difficult to interpret. Alternatively, programs like Caleb [10] and SimPed [11] are limited in the sense that Caleb cannot handle arbitrary family structures, and SimPed cannot accurately or easily model the LD patterns that typically arise in a genome-wide linkage study. Lastly, the most common approach, which avoids LD by using a sparsely spaced subset of the original dense single nucleotide polymorphisms (SNPs) [1214], can restore (or nearly restore) control over the type 1 error, but some amount of power [15] is inevitably lost.

In contrast to these simulation programs, Haplodrop can easily and adequately model realistic patterns of LD without imposing constraints on the family structure. Haplodrop facilitates the accurate estimation of the null distribution of any multipoint linkage statistic in the absence or presence of LD, which should help researchers control the type 1 error and estimate accurate $p$ values from dense SNP linkage data. In addition, Haplodrop has user-friendly options for masking genotype data and is freely available as part of the EAGLET genetic software package (www.mathmed.org/wclstewart/SOFT/EAGLET/eaglet.html).

\section{Materials and Methods}

Haplodrop uses a Markov model to describe the correlation of alleles along the chromosome [16] and a multilocus model of inheritance to segregate the haplotypes of founders to all nonfounders in a family. This permits the rapid generation of multilocus genotype configurations that reflect realistic patterns of LD. Furthermore, since each replicate is generated under the null hypothesis of no linkage, an accurate estimate of the null distribution (as well as the p value) of any linkage statistic of interest (e.g. the average maximum LOD [15], the maximum multipoint LOD [17], the maximized multipoint LOD [18], or the Kong and Cox LOD [6]) is easily obtained.

For a given family with $N$ founders, let $\left(\boldsymbol{H}_{1}{ }^{(i)}, \boldsymbol{H}_{2}{ }^{(i)}\right)$ denote the pair of paternal and maternal $n$-site haplotypes of the $i$ th founder, for $i=1, \ldots, N$. The $n$ components of $\boldsymbol{H}_{j}^{(i)}$ are indicator variables $\left(A_{j, 1}{ }^{(i)}, \ldots, A_{j, n^{(i)}}\right)$, where each $A_{j, k}{ }^{(i)}$ is 0 or 1 depending on the allele at site $k$ in haplotype $\boldsymbol{H}_{j}^{(i)}$. Haplodrop assumes that for each haplotype in each founder the indicator variables follow a firstorder Markov model with transition probabilities $t_{u, v}(m)=\operatorname{Pr}\left(A_{j}\right.$, $\left.(m+1)^{(i)}=v \mid A_{j, m}{ }^{(i)}=u\right)$ for $m=1, \ldots,(n-1)$. In particular,

$$
\begin{aligned}
t_{u, u}(m) & =\operatorname{Pr}\left(A_{j,(m+1)}{ }^{(i)}=u \mid A_{\left.j, m^{(i)}=u\right)}\right. \\
& =\operatorname{Pr}\left(A_{j,(m+1)}{ }^{(i)}=u, A_{j, m}{ }^{(i)}=u\right) / \operatorname{Pr}\left(A_{j, m}{ }^{(i)}=u\right) \\
& =\left(\mathrm{LD}_{m,(m+1)} / p_{u}\right)+q_{u}
\end{aligned}
$$

where $p_{u}=\operatorname{Pr}\left(A_{j, m}{ }^{(i)}=u\right), q_{u}=\operatorname{Pr}\left(A_{j,(m+1)}{ }^{(i)}=u\right)$ and $\operatorname{LD}_{m,(m+1)}=$ $\operatorname{Pr}\left(A_{j,(m+1)}{ }^{(i)}=u, A_{j, m}{ }^{(i)}=u\right)-p_{u} q_{u}$. Thus, the transition rate (and hence, the haplotype diversity in the simulated data) is directly influenced by the magnitude of $\operatorname{LD}_{m,(m+1)}$. Furthermore, all four transition probabilities are completely determined by the pair $t_{u \text {, }}$ ${ }_{u}(m)$ since $t_{u,(1-u)}(m)=1-t_{u, u}(m)$.

Once haplotypes have been generated for every founder, Haplodrop segregates these haplotypes to all non-founders using the Haldane model [19] of multilocus inheritance to allow for recombination. If desired, the simulated genotypes for selected family members may be masked. Haplodrop repeats this process for each family, which results in a multilocus genotypic configuration for the entire data set realized from the null distribution of no linkage, but in the presence of some pre-specified level of LD. Thus, the only required inputs for Haplodrop are: a pedigree structure, a genetic map, the number of replicates to generate, a vector of pairwise $r^{2}$ values for adjacent SNPs, and their corresponding allele frequencies. To simulate haplotypes that preserve the complete correlation structure and not just the pairwise LD, the haplotypes of founders can also be sampled with replacement from a set of reference haplotypes (e.g. HapMap). Given the widespread availability of high-quality reference data sets, and given the wide range of excellent haplotype imputation programs, we believe that this nonparametric sampling alternative is an excellent way to capture realistic patterns of fine-scale LD.

\section{Data Description}

To illustrate in a realistic setting the negative effect of ignoring $\mathrm{LD}$, and to demonstrate the validity of Haplodrop on multiple levels, we did the following. First, we used Haplodrop to simulate 5,000 replicate sets of linkage data 
Fig. 1. The cumulative distribution functions for the maximum Kong and Cox LOD with LD (dashed) and without LD (solid) are shown $\mathbf{a}, \mathbf{b}$. The critical values for testing at the 5\% level are indicated by the vertical dotted lines. Calculations are based on 5,000 replicates with 100 ASPs and 100 ASTs per replicate, respectively.

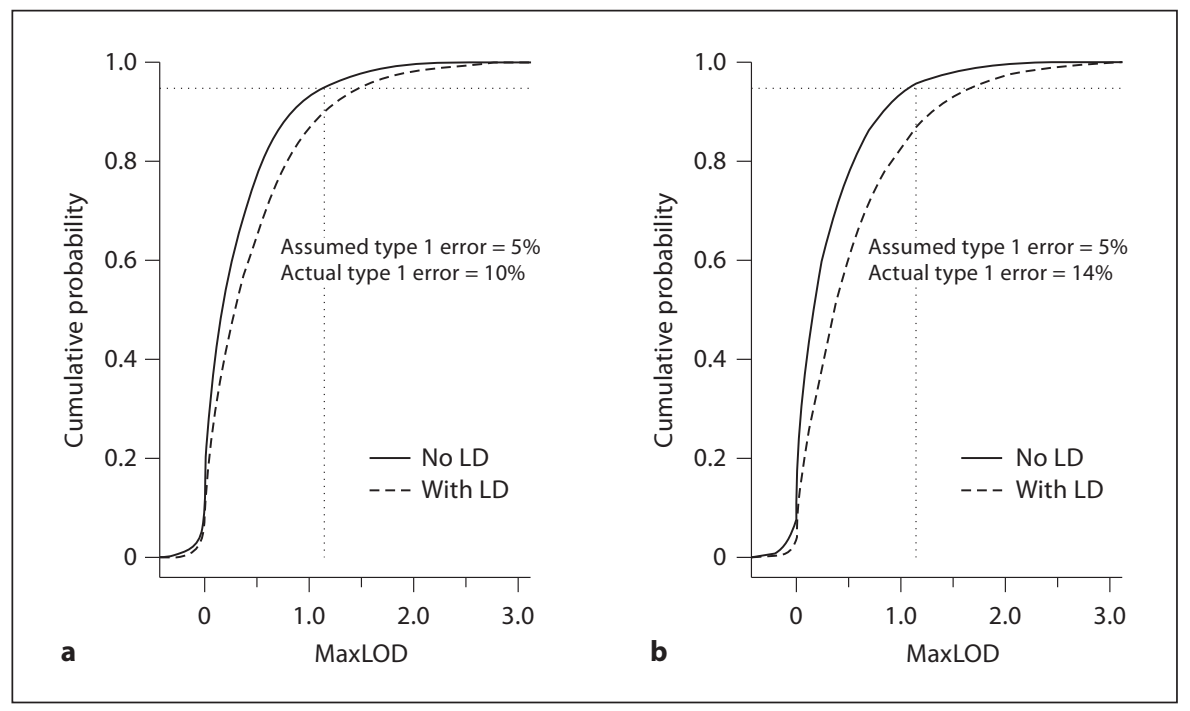

with 100 ASPs and 100 affected sibling trios (ASTs) per replicate, under the null hypothesis of no linkage, but with and without LD. When the simulation involved LD, the pattern was estimated from the pairwise LD of 50 SNPs of a real dense SNP linkage scan [20]. The SNPs were spaced approximately $1 \mathrm{cM}$ apart. Second, using the same LD pattern, we used Haplodrop to simulate linkage data under the null hypothesis of no linkage with 12, 24, 48, 120, 240, 600 , and 1,200 ASPs; and with 8, 16, 32, 80, 160, 400, and 800 ASTs. In this way, the total number of genotyped individuals across ASP and AST designs remains constant for each point in the sequence. To assess the discrepancy between the maximum likelihood estimate (MLE) of LD and the true $\mathrm{LD}$, we computed the average absolute error across all 49 contiguous intervals. Third, we used Haplodrop and SimPed to simulate 1,500 replicates under the null hypothesis of no linkage, but with a common pattern of LD, where each replicate contained 250 ASPs. The common pattern of LD used 8 equi-frequent SNPs that were spaced $5.26 \mathrm{cM}$ apart with a constant pairwise $r^{2}$ of 0.25 .

\section{Results}

Our user-friendly and well-documented simulation program, Haplodrop, generates dense SNP data under the null hypothesis of no linkage, while allowing for a range of different LD patterns (which includes the special case of no LD). Figure la, $b$ shows the distribution functions of the maximum Kong and Cox LOD for 100 ASPs and 100 ASTs under the null hypothesis of no linkage, but

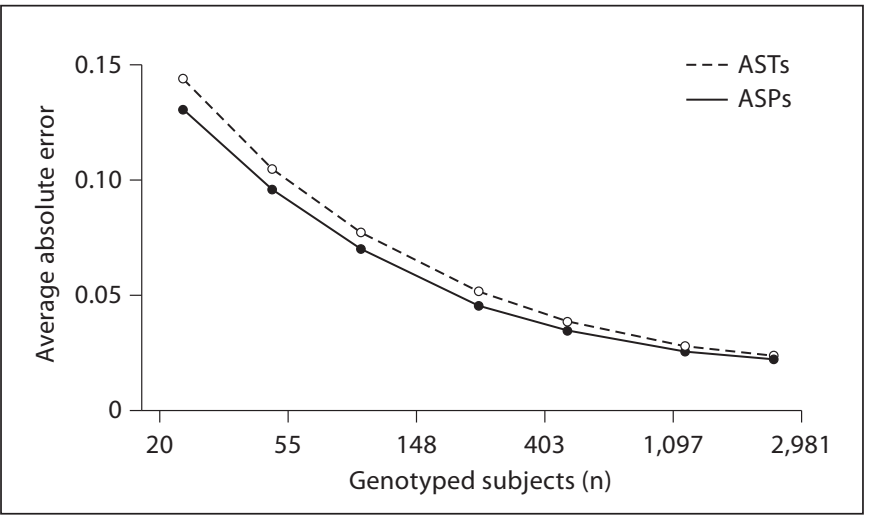

Fig. 2. For each design, ASPs (solid) and ASTs (dotted), the total number of genotyped individuals was held constant at 24, 48, 96, $240,480,1,200$, and 2,400 subjects, respectively. Then, for each point on the plot, the absolute error between the true LD and the MLE of LD was averaged over all 49 intervals and over 100 replicates. The true LD pattern was estimated from a real dense SNP linkage scan, and the $\mathrm{x}$-axis is plotted on the logarithmic scale.

with and without LD. For the distribution functions that involve $\mathrm{LD}$, the pattern of $\mathrm{LD}$ was estimated from a real dense SNP linkage scan. From figure 1a, we see that when $\mathrm{LD}$ is ignored, the type 1 error increases from 5 to $10 \%$ for ASPs, and from figure $1 \mathrm{~b}$, the type 1 error increases from 5 to $14 \%$. This shows that naturally occurring patterns of LD can dramatically increase the type 1 error of a modern linkage scan.

From figure 2, we see that the average absolute error between the MLE of LD and the true pairwise LD ap- 
proaches zero as the total number of genotyped subjects increases. The apparent convergence of the average absolute error suggests that Haplodrop correctly generates dense SNP data with any pre-specified level of LD. In addition, figure 2 also shows that when SNPs are separated by about $1 \mathrm{cM}$, as few as $400-600$ genotyped individuals are enough to accurately estimate the true underlying pattern of LD.

To further validate Haplodrop in the context of linkage, we used Haplodrop and SimPed to generate multipoint SNP data for ASPs under the null hypothesis of no linkage, but with a common pattern of LD. The common pattern of LD used 8 equi-frequent SNPs with a constant level of LD between adjacent SNPs. We used a Kolmogorov-Smirnov test to compare the null distributions of the maximum LOD. For each program (Haplodrop and SimPed), the null distributions were estimated from 1,000 replicates with 250 ASPs per replicate. The $p$ value (0.314) of the Kolmogorov-Smirnov test is not significant, which suggests that (as expected) the two programs are in fact simulating multipoint SNP data from the same distribution.

\section{Discussion}

Relative to existing programs [8-11], Haplodrop is an attractive alternative that gives researchers the ability to estimate accurate $p$ values and control the type 1 error of their multipoint linkage study in the presence of LD. This is important for two reasons. First, for the most commonly used approach (i.e. the approach that assumes the absence of LD between sparsely spaced SNPs), inflation of the type 1 error can be severe, especially when families are enriched for affecteds and there is missing parental information. Second, since the null distributions of many commonly used multipoint linkage statistics (e.g. maxLOD, maxHLOD, etc.) are unknown, the must be estimated empirically through simulation. In particular, the current practice of routinely using different thresholds for the same multipoint linkage statistic is unsatisfactory. Furthermore, by controlling the type 1 error in the presence of LD, thousands of clinically unimportant mutations may be excluded from further study, which could greatly improve the overall efficacy of targeted next-generation sequencing.

Note that in addition to controlling the type 1 error, Haplodrop also scales easily to whole-genome dense SNP arrays. For instance, to simulate 100 replicates of multilocus genotype data at all 11,964 SNPs of chromosome 20, with $100 \mathrm{sib}$ pairs per replicate, on a $3.2 \mathrm{GHz}$ machine with $2,000 \mathrm{Mb}$ of memory takes less than $90 \mathrm{~min}$. Furthermore, Haplodrop is written entirely in Perl, which makes it portable across computing platforms and available to a wide range of researchers.

Given the current wealth of computing power, and given that the null distributions for most multipoint linkage statistics are unknown, we submit that researchers should routinely use Haplodrop to estimate the $p$ values of their linkage studies. A simple and straightforward procedure for estimating the genome-wide $p$ value is to correct the chromosome-specific p value (provided by Haplodrop) for the number of independent autosomes that are tested. With this approach, researchers can control the type 1 error of their genome-wide linkage scans irrespective of the particular test statistic that they choose to use. Moreover, we plan to fully integrate Haplodrop within the EAGLET suite of genetic analysis programs so that researchers can easily compute $p$ values for a wide range of powerful linkage statistics, including those that extract the maximum amount of information from dense SNP data $[15,21]$.

\section{Acknowledgements}

We would like to thank the Reviewers for their thoughtful comments and remarks. This work was supported by the National Institutes of Health: NARSAD 17832, MH65213, NS070323, MH65213 and NS061829-02 S1.

\section{References}

Type 1 Error Control in Linkage Analysis 
4 Huang Q, Shete S, Amos CI: Ignoring linkage disequilibrium among tightly linked markers induces false-positive evidence of linkage for affected sib pair analysis. Am J Hum Genet 2004;75:1106-1112.

$\checkmark 5$ Lander E, Kruglyak L: Genetic dissection of complex traits: guidelines for interpreting and reporting linkage results. Nat Genet 1995;11:241-247.

$\checkmark 6$ Kong A, Cox NJ: Allele-sharing models: LOD scores and accurate linkage tests. Am J Hum Genet 1997;61:1179-1188.

7 Abecasis GR, Wigginton JE: Handling marker-marker linkage disequilibrium: pedigree analysis with clustered markers. Am J Hum Genet 2005;77:754-767.

$\checkmark 8$ Thomas A: Estimation of graphical models whose conditional independence graphs are interval graphs and its application to modeling linkage disequilibrium. Comput Stat Data Anal 2009;53:1818-1828.

-9 Thomas A: Assessment of SNP streak statistics using gene drop simulation with linkage disequilibrium. Genet Epidemiol 2010;34: 119-124.
Greenberg DA: Computer simulation is an undervalued tool for genetic analysis: a his torical view and presentation of SHIMSHON - a Web-based genetic simulation package. Hum Hered 2011;72:247-257.

11 Leal SM, Yan K, Muller-Myhsok B: Simped: a simulation program to generate haplotype and genotype data for pedigree structures. Hum Hered 2005;60:119-122.

12 Bellenguez C, Ober C, Bourgain C: Linkage analysis with dense SNP maps in isolated populations. Hum Hered 2009;68:87-97.

13 Evans DM, Cardon LR: Guidelines for genotyping in genomewide linkage studies: single-nucleotide-polymorphism maps versus microsatellite maps. Am J Hum Genet 2004; 75:687-692.

14 Webb EL, Sellick GS, Houlston RS: Snplink: Multipoint linkage analysis of densely distributed SNP data incorporating automated linkage disequilibrium removal. Bioinformatics 2005;21:3060-3061.

15 Stewart WC, Drill EN, Greenberg DA: Finding disease genes: a fast and flexible approach for analyzing high-throughput data. Eur J Hum Genet 2011;19:1090-1094.

16 Bercovici S, Meek C, Wexler Y, Geiger D: Estimating genome-wide IBD sharing from SNP data via an efficient hidden Markov model of LD with application to gene mapping. Bioinformatics 2010;26:i175-i182.
7 Lathrop GM, Lalouel JM, Julier C, Ott J: Strategies for multilocus linkage analysis in humans. Proc Natl Acad Sci USA 1984;81: 3443-3446.

18 Hodge SE, Elston RC: Lods, wrods, and mods: the interpretation of lod scores calculated under different models. Genet Epidemiol 1994;11:329-342.

19 Haldane JBS: The combination of linkage values and the calculation of distances between the loci of linked factors. J Genet 1919; 8:299-309.

20 Rodriguez-Murillo L, Subaran R, Stewart WC, Pramanik S, Marathe S, Barst RJ, Chung WK, Greenberg DA: Novel loci interacting epistatically with bone morphogenetic protein receptor 2 cause familial pulmonary arterial hypertension. J Heart Lung Transplant 2010;29:174-180.

21 Stewart WC, Peljto AL, Greenberg DA: Multiple subsampling of dense SNP data localizes disease genes with increased precision. Hum Hered 2010;69:152-159. 